\title{
Relation between boundary slip mechanisms and waterlike fluid behavior
}

\author{
Patricia Ternes, ${ }^{1, *}$ Evy Salcedo, ${ }^{2}$ and Marcia C. Barbosa ${ }^{1}$ \\ ${ }^{1}$ Instituto de Física, Universidade Federal do Rio Grande do Sul, Caixa Postal 15051, 91501-970 Porto Alegre, RS, Brazil \\ ${ }^{2}$ Coordenadoria Especial de Física, Química e Matemática, Universidade Federal de Santa Catarina, \\ Rua Pedro João Pereira 150, 88905-120 Araranguá, SC, Brazil
}

(Received 12 January 2018; published 12 March 2018)

\begin{abstract}
The slip of a fluid layer in contact with a solid confining surface is investigated for different temperatures and densities using molecular dynamic simulations. We show that for an anomalous waterlike fluid the slip goes as follows: for low levels of shear, defect slip appears and is related to the particle exchange between the fluid layers; at high levels of shear, global slip occurs and is related to the homogeneous distribution of the fluid in the confining surfaces. The oscillations in the transition velocity from defect to global slip are shown to be associated with changes in the layering distribution in the anomalous fluid.
\end{abstract}

DOI: 10.1103/PhysRevE.97.033104

\section{INTRODUCTION}

The no-slip condition is the assumption that the fluid velocity is zero when in contact with the solid confined geometry. For a macroscopic flow this is a trustworthy boundary condition, and it is fundamental for the continuum theory validity. For confined geometries the hydrodynamic equations are no longer valid, and in this case, the use of the no-slip boundary condition is at least questionable. Many experimental [1-8], theoretical, and computational [9-15] results report that there are several flow boundary conditions consistent with the fluid behavior and mobility [16-19] beyond the no-slip boundary condition. The amount of slip is usually measured through the magnitude of the slip length, defined as the ratio between the shear rate and the slip velocity [16-19]. For most liquids the slip length increases with the shear rate and stabilizes at $v_{0}$ [19]. Its value, however, depends on the thickness of the confining system in a nontrivial way. For apolar materials, such as hexane [20] and $n$-decane [17,19], the slip length increases with the film thickness. However, for polyamide-6,6 [21] and for water [22] the slip length decreases with an increase in the film thickness. Complementarily, the behavior of the shear viscosity gives the slip length. For $n$-decane the viscosity increases with an increase in the film thickness [23], while for polyamide-6,6 [21] the viscosity decreases with an increase in the film.

For water the situation is even more complex. Confined water in microchannels presents a slip length of the order of nanometers [24-28] and therefore no-slip boundary conditions are not applicable. As the channel size decreases, the water mobility increases [29-32]. The slip length for nanochannels becomes of the order of micrometers, which implies that the use of no-slip boundary conditions could be problematic.

Even though the qualitative behavior of the slip length is known, the specific value of the slip length is widely scattered. In the particular case of water, it depends on the surface energy and roughness and the fluid temperature and density

\footnotetext{
*patricia.terdal@gmail.com
}

[26,33-42]. Thus, a strategy for understanding this qualitative behavior is to explore the mechanism behind the change of the slip with the shear rate, whether the slip occurs through one single process or whether it involves a number of steps which depend on the velocity. In the case of apolar Lennard-Jones (LJ) fluids the slip changes through two mechanisms: defect slip and global slip [16-19]. The transition from defect to global slip occurs at a shear rate $v_{0}$. Therefore for a given fluid and wall, the slip length depends on the temperature and density from the behavior of $v_{0}$. Unfortunately, very little is known about the behavior of $v_{0}$.

Here we add another component to this already complex problem. We explore the mechanism behind liquid slip in the case of anomalous waterlike fluids. An anomalous fluid is characterized by having a maximum in the density versus temperature at fixed pressure and a maximum and a minimum in the diffusion coefficient versus pressure at constant temperature [43-48]. Under high confinement, these fluids exhibit additional anomalous behaviors and new phases $[49,50]$. When an anomalous fluid is nanoconfined the thermodynamic and dynamic properties differ from the properties observed in the bulk [29-31,51]. For instance, for a bulk system the fluid is described as homogeneously distributed. This is not the case for confined systems. A waterlike fluid forms layers which depend on the film thickness $[49,50,52-55]$. Due to the layering, particles show different behaviors in different layers, which allows for the anomalous flux observed in confined waterlike materials.

In this paper we investigate the slip mechanism of a waterlike fluid. After testing the fluid for the defect slip and global slip transition at $v_{0}$, we study the connection between the behavior of $v_{0}$ at different temperatures and densities and the structure and dynamics of the layers.

A waterlike fluid is modeled by an effective potential with two length scales separated by an energy barrier. The use of an effective potential allows us to explore a large portion of the temperature-versus-density phase diagram. Molecular dynamics simulations of the planar Couette flow for this anomalous fluid test the presence of defect and global slip at 


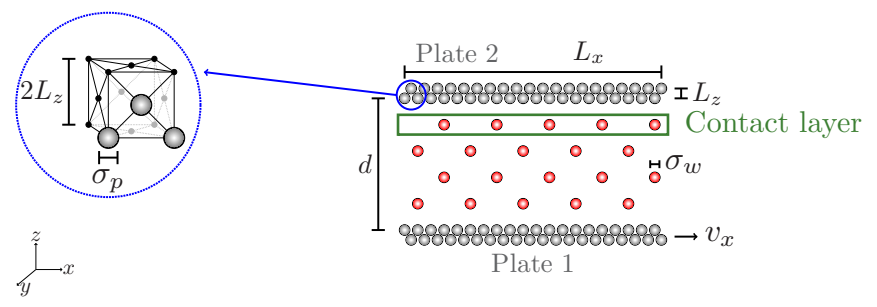

FIG. 1. Schematic of the waterlike fluid confined within parallel plates. The $y$ direction is omitted.

different densities and temperatures. This paper is organized as follows: Section II presents the model and simulation details, Sec. III reports the results, and Sec. IV gives the conclusions.

\section{THE MODEL, SIMULATION, AND METHODS}

\section{A. The model}

A waterlike fluid is confined in the planar Couette geometry shown in Fig. 1. Each plate is formed by $N_{p}=676$ spherical particles of diameter $\sigma_{p}$ organized in two planar layers, forming a face-centered cubic lattice, with $L_{x}=L_{y}=20.2 \sigma_{p}$ and $L_{z}=0.7 \sigma_{p}$, as shown in Fig. 1 . The separation between the plates, or channel height, is $d$. The liquid is sheared by moving the bottom bounding wall (plate 1 in Fig. 1) at speed $v_{x}$, while the top bounding wall (plate 2 in Fig. 1) is held fixed. The contact layer is composed of fluid particles whose centers of mass lie between plate 2 and the first minimum in the density profile.

The particles at the plates are tethered to its lattice site by a linear spring with constant $k^{*}=k\left[\sigma_{w}^{2} /(m \varepsilon)\right]^{1 / 2}=50$ and characteristic excursion $\xi=\sqrt{k_{B} T / k}$ [56], where $k_{B}$ is the Boltzmann constant, and $T$ is the plate temperature. The particles at the plates also interact with each other via a standard LJ 12-6 potential with $\varepsilon$ depth and $\sigma_{p}$ [57,58]. The fluid is modeled by $N_{w}=500$ identical waterlike particles with diameter $\sigma_{w}=\sigma_{p}$. The fluid particles interact through a core-softened potential given by $[46,47]$

$$
\begin{aligned}
\frac{U_{w}\left(r_{i j}\right)}{\varepsilon}= & 4\left[\left(\frac{\sigma_{w}}{r_{i j}}\right)^{12}-\left(\frac{\sigma_{w}}{r_{i j}}\right)^{6}\right] \\
& +u_{0} \exp \left[-\frac{1}{c_{0}^{2}}\left(\frac{r_{i j}-r_{0}}{\sigma_{w}}\right)^{2}\right] .
\end{aligned}
$$

This potential presents two length scales: a standard LJ 12-6 potential plus a Gaussian centered at $r_{0}$, with width $c_{0}$ and depth $u_{0}$, where $r_{i j}=\left|\vec{r}_{i}-\vec{r}_{j}\right|$ is the distance between fluid particle $i$ and particle $j[46,47]$. With variation of the parameters $u_{0}, c_{0}, r_{0}$, and $\sigma_{w}$ this potential can represent a whole family of intermolecular interactions. In this work the chosen parameters are $u_{0}=5.0, c_{0}=1.0$, and $r_{0}=0.7 \sigma_{w}$. For these parameters the potential presents one scale at $r_{i j} \approx 1.2 \sigma_{w}$ and the other scale at $r_{i j} \approx 2 \sigma_{w}$, each scale being related to the interaction between two water tetramer clusters, as shown in

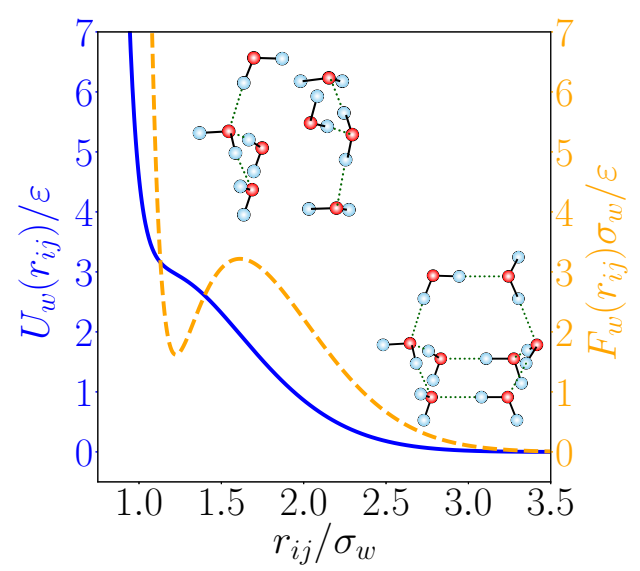

FIG. 2. Left axis: Isotropic effective potential as a function of the particle separation (solid blue line). Right axis: Force related to the effective potential as a function of the particle separation (dashed orange line).

the illustration of the force in Fig. 2 [59,60]. The bulk system of spherical particles interacting through this potential exhibits diffusion, structural, and density anomalous behavior observed also in bulk water $[46,47]$.

The particles of this waterlike fluid interact with the wall particles through the purely repulsive potential given by the Weeks-Chandler-Andersen LJ potential $[61,62]$,

$$
U_{w p}\left(r_{i j}\right)= \begin{cases}U_{\mathrm{LJ}}\left(r_{i j}\right)-U_{\mathrm{LJ}}\left(r_{c}\right), & r_{i j} \leqslant r_{c}, \\ 0, & r_{i j}>r_{c},\end{cases}
$$

where $U_{\mathrm{LJ}}$ is the standard 12-6 LJ and $r_{c}$ is the cutoff distance $\left(r_{c}=2^{1 / 6} \sigma_{w p}\right)$. The effect radius, $\sigma_{w p}$, is determined through the Lorentz-Berthelot mixing rule $\left[\sigma_{w p}=\left(\sigma_{p}+\sigma_{w}\right) / 2\right]$ and is used when one fluid particle is interacting with one wall particle [63]. The repulsive fluid-plate interaction causes an excluded volume, therefore the fluid effective density will be $\rho=N_{w} /\left[\left(d-\sigma_{w p}\right) L_{x} L_{y}\right][64,65]$.

\section{B. The simulations}

The system was studied by molecular dynamics simulations at constant $N V T$ using a homemade program. A Nosé-Hoover heat bath with coupling parameter $Q=2$ was applied to the plate's particles in order to maintain the fixed temperature $[66,67]$. The system was analyzed at different densities and heat bath temperatures. The temperature varied from $T^{*}=$ $k_{B} T / \varepsilon=0.025$ up to $T^{*}=0.650$, and $\xi$ varied from 0.022 up to 0.110 . $N$ was fixed and the density decreased by increasing the distance between the plates from $d^{*}=d / \sigma_{w}=3.8$ to $d^{*}=$ 9.8. The initial configuration of the confined fluid was set on the solid state and, without shear $\left(\vec{v}_{x}=0\right)$, further equilibrated over $5 \times 10^{5}$ steps. Then, in order to obtain the temperatureversus-density phase diagram of the confined system, $2 \times 10^{6}$ steps were performed. The transversal pressure, $P$, is computed analogously to the bulk pressure [68],

$$
P=\rho k_{B} T+\frac{1}{V}\left\langle v_{\perp}\right\rangle
$$


where $v_{\perp}$ is the transversal virial expression,

$$
v_{\perp}=-\sum_{i} \sum_{j>i} \frac{z_{i j}^{2}}{r_{i j}}\left(\frac{\partial U_{w}\left(r_{i j}\right)}{\partial r_{i j}}\right) .
$$

Next, the bottom bounding wall moves at a constant speed $\vec{v}_{x}$. At each density and temperature, several simulations with wall velocities varying from low shear levels, $v_{x}^{*}=$ $v_{x}(m / \varepsilon)^{1 / 2}=0.001$, up to high shear levels, $v_{x}^{*}=15.0$ (where the bottom wall velocity is about five times higher than the fluid thermal velocity), were carried out. The fluid heats up due to shear and the system reaches a new equilibrium temperature after $3 \times 10^{5}$ steps. Since the equilibrium temperature of the fluid depends on the shear level, the temperature used in the graphs is the heat bath obtained from the thermostat fixed at the wall. After equilibration, an additional $8 \times 10^{6}$ steps were performed to store physical quantities for the system with shear. The structure of the waterlike fluid in the contact layer was analyzed through the parallel radial distribution function, $g_{\|}\left(r_{x y}\right)$. This distribution function is defined as [64]

$$
\begin{aligned}
g_{\|}\left(r_{x y}\right) \equiv & \frac{1}{\rho^{2} V} \sum_{i \neq j} \delta\left(r_{x y}-r_{i j}\right)\left[\theta\left(\left|z_{i}-z_{j}\right|\right)\right. \\
& \left.-\theta\left(\left|z_{i}-z_{j}\right|-\delta z\right)\right],
\end{aligned}
$$

where $r_{x y}$ is the parallel distance between particles, and $\theta(z)$ is the Heaviside function, which limits the particle sum in a layer of thickness $\delta z$. The fluid structure was also analyzed through the translational order parameter, defined as [69]

$$
t \equiv \int_{0}^{\zeta_{c}}\left|g_{\|}(\zeta)-1\right| d \zeta
$$

where $\zeta_{c}=0.5 L_{x} \rho_{l}^{1 / 2}$ is the cutoff distance set to half the simulation box times the density of the contact layer, and $\zeta=$ $r_{x y}\left(\rho_{l}\right)^{1 / 2}$ is the distance $r_{x y}$ in units of the mean interparticle separation in the parallel direction. The translational order parameter measures how the system is structured. For an ideal gas $t=0$, and for more structured phases $t$ increases.

The equations of motion were integrated with a time step $\delta t^{*}=\delta t\left[\varepsilon /\left(m \sigma_{w}^{2}\right)\right]^{1 / 2}=0.0025$, and five independent runs were used to evaluate the confined anomalous fluid properties. All quantities are given in LJ units [63], and for simplicity, the superscript asterisk employed in dimensionless quantities is excluded.

\section{The slip boundary conditions}

Usually confined systems are analyzed employing no-slip boundary conditions under which the mean velocity of the fluid particles in the contact layer is 0 . Even though the no-slip boundary condition is good to describe confinement up to microchannels [24-28], this might not be the case for nanoconfined geometries [29-32]. Different slip condition mechanisms might occur as the relative velocity between the fluid and the wall is changed. For planar Couette flow two boundary slip mechanisms are predicted for nonanomalous fluid: defect slip and global slip [16-19]. Defect slip depends on the local and ordered hops of the fluid particles in the contact layer. These hops occur due to the presence of disorder in the ground state of the wall-fluid interaction, which obeys
Arrhenius dynamics. Global slip occurs when all fluid particles in the contact layer are detached from the wall by movement.

It is possible to verify the occurrence of these boundary slip mechanisms by analyzing the average particle motion. Under the no-slip condition the particles oscillate around the minimum of the ground state of the particle-wall interaction. In this case the fluid particles in the contact layer have no preferential direction of movement. For the slip condition the movement of fluid particles in the contact layer is in the driven direction. Thus, to compute this move, we compute the probability of one particle's moving in the driven direction, $P_{\mathrm{DD}}$, defined as

$$
P_{\mathrm{DD}}=\frac{100}{S} \sum_{i=1}^{S}\left[\frac{\sum_{j=1}^{N_{\mathrm{CL}}}\left[x_{j}(i)-x_{j}(i-1)\right] \sigma_{i j}}{\sum_{j=1}^{N_{\mathrm{CL}}}\left|x_{j}(i)-x_{j}(i-1)\right|}\right],
$$

where $S$ is the number of simulation steps, $N_{\mathrm{CL}}$ is the number of fluid particles in the contact layer, $x_{j}(i)-x_{j}(i-1)$ is the displacement of particle $j$ between step $i-1$ and step $i$, and $\sigma_{i j}$ is a piecewise function. If the displacement is in the driven direction $\left[x_{j}(i)-x_{j}(i-1) \geqslant 0\right]$, then $\sigma_{i j}=1$, and if the displacement is in the opposite direction $\left[x_{j}(i)-x_{j}(i-1)<0\right]$, then $\sigma_{i j}=0$. If $P_{\mathrm{DD}}$ is close to $50 \%$ and the contact layer is stationary, the no-slip boundary conditions are valid. As the fluid particles hop from one site to another, $P_{\mathrm{DD}}>50 \%$, the system is in the defect slip boundary condition and the particles move in one direction. For $P_{\mathrm{DD}}$ close to $100 \%$ the particles in the contact layer move in the driven direction and the system is under the global slip boundary condition. Then the transition from the no-slip condition to the defect and global slip conditions is identified by the transition of the logistic function

$$
P_{\mathrm{DD}}=100-\frac{50}{1+\left(v_{x} / v_{0}\right)^{\alpha}},
$$

where $\alpha$ is the steepness of the curve, which is related to the necessary velocity increases to promote the transition from defect slip to global slip, and $v_{0}$ is the logistic midpoint, which is related to the bottom wall velocity that promotes global slip.

\section{RESULTS AND DISCUSSION}

In order to understand the effect of different boundary conditions on the behavior of the waterlike fluid, first we obtained the behavior of the system under the no-slip boundary condition (without shear). For this system, the pressure-versusdensity phase diagram presents isochores monotonic at a temperature above $T^{*}=0.400$. Below $T^{*}=0.400$ van der Waals loops indicate coexistence between the two phases. The coexistence densities were then obtained using the Maxwell construction, and the critical points are given by $d^{2} P / d \rho^{2}=0$. The temperature-versus-density phase diagram in Fig. 3 summarizes this information. The regions inside the curves in Fig. 3 represent the coexistence between the various two-dimensional liquid, liquid crystal, and crystal phases [50]. Open symbols in Fig. 3 show the critical points. For zero shear the phase diagrams obtained using the thermostat on the wall or in the fluid are equal [50].

A confined waterlike system is characterized by the presence of planar layers. The number of layers depends on the 


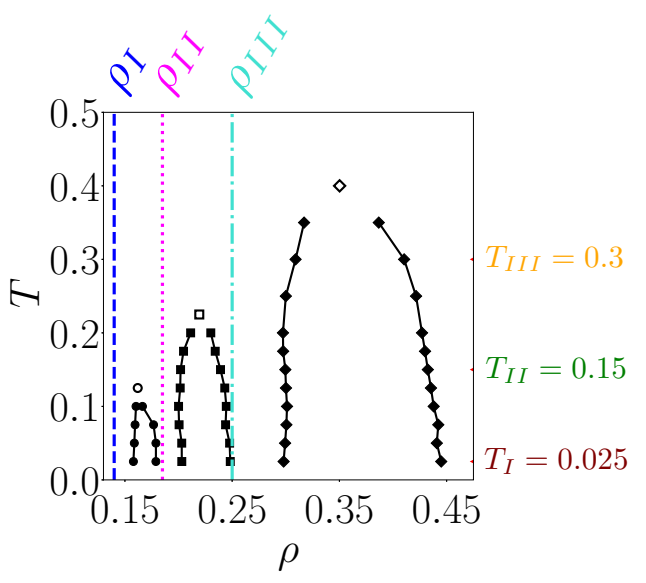

FIG. 3. Temperature-versus-density phase diagram without shear. Solid black lines represent regions of first-order phase transitions that end in three critical points (open symbols). The temperatures $T_{\mathrm{I}}, T_{\mathrm{II}}$, and $T_{\mathrm{III}}$ and densities $\rho_{\mathrm{I}}, \rho_{\mathrm{II}}$, and $\rho_{\mathrm{III}}$ indicate the values used in the following results

film thickness, as illustrated in Fig. 4. Since in our system the number of particles is kept fixed, the change in the film thickness is equal to the change in the density. For temperatures from $T=0.025$ to $T=0.65$ and for density $\rho_{\mathrm{I}}=0.14$ (dashed line in Fig. 3), the fluid forms five layers; for density $\rho_{\mathrm{II}}=0.18$ (dotted line in Fig. 3), the fluid is structured in four layers; and for density $\rho_{\mathrm{III}}=0.25$ (dot-dashed line in Fig. 3), the fluid forms three layers.

We also explored the effect of different boundary conditions. In particular, we studied the behavior of the fluid as the bottom wall moves for systems with different fluid densities and several thermal bath temperatures. Figure 5 illustrates the probability that a particle will move in the driven direction as a function of the bottom wall speed. We identified three characteristic regions. The no-slip condition is valid for low bottom wall velocities; $50 \%$ of the particles move in the driven direction, while the other $50 \%$ move in the opposite direction. At temperatures of $T_{\mathrm{I}}=0.025$ and $\rho_{\mathrm{I}}=0.14$, the no-slip condition is valid for velocities up to $v_{x}<2$. As the bottom wall velocity increases, $P_{\mathrm{DD}}$ increases, leading to the defect slip condition, in which a few more particles move in the same direction as the bottom wall. As $v_{x}$ increases even further, the system reaches the global slip condition when $v_{x}>v_{0}$, which implies that most fluid particles in the contact layer move in the same direction as the bottom wall. The transition between the boundary conditions is characterized by the logistic equation [Eq. (8)] illustrated by the solid line in Fig. 5. For $T_{\mathrm{I}}$ and $\rho_{\mathrm{I}}$, the

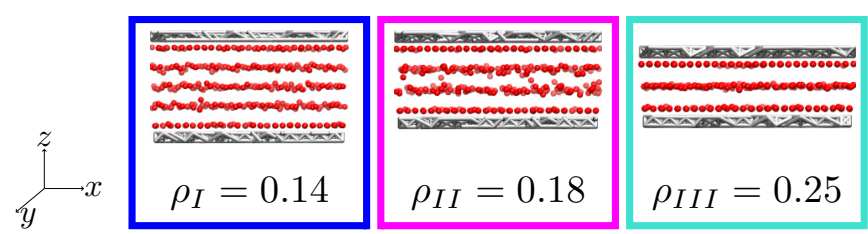

FIG. 4. Snapshots of a waterlike fluid confined between parallel plates, without shear, at $T_{\mathrm{I}}=0.025$. To simplify viewing, the $y$ direction is omitted.

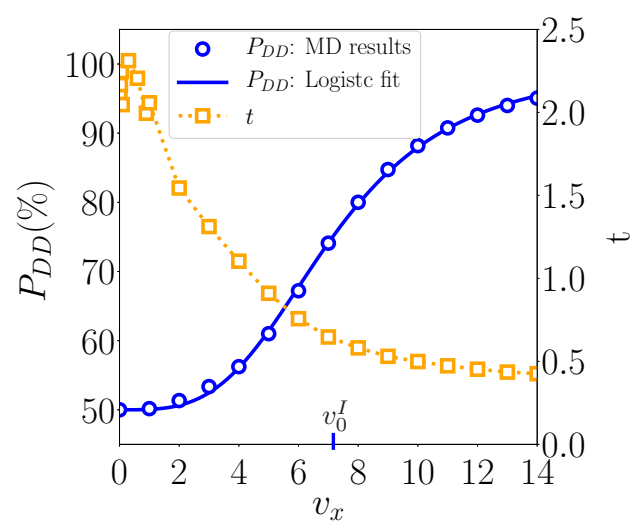

FIG. 5. Left axis: Probability that the particles in the contact layer will move in the driven direction as a function of the bottom wall velocity. Blue circles represent results from simulations for thermal bath temperature $T_{\mathrm{I}}=0.025$ and fluid density $\rho_{\mathrm{I}}=0.14$. The solid blue line is a logistic fit. Right axis: Translational order parameter as a function of the bottom wall velocity for $\rho_{\mathrm{I}}$ at $T_{\mathrm{I}}$.

logistic fit is given by $v_{0}^{\mathrm{I}}=7.15(4)$ and $\alpha^{\mathrm{I}}=3.39(7)$. A similar graph is also observed for higher densities and temperatures. This behavior was also observed for a Lennard-Jones-like fluid for one specific temperature and density [19].

In order to understand how the slip condition is affected by the thermodynamic state of the anomalous fluid, the behavior of $v_{0}$ and $\alpha$ is analyzed at different temperatures and densities (in our system this implies the thickness of the film). Figure 6 shows the behavior of the logistic midpoint, $v_{0}$, defined by Eq. (8), as a function of the density for distinct temperatures indicated in Fig. 3 ( $T_{\mathrm{I}}, T_{\mathrm{II}}$, and $\left.T_{\mathrm{III}}\right)$. This result shows that $v_{0}$ increases with the density at a fixed temperature.

At low temperatures the particles at a constant density are more structured and a higher kinetic energy, larger $v_{0}$, from the moving wall would be needed for the transition to global slip. Similarly, at a constant temperature, as the system becomes denser, it is also more structured [50] and, therefore, would

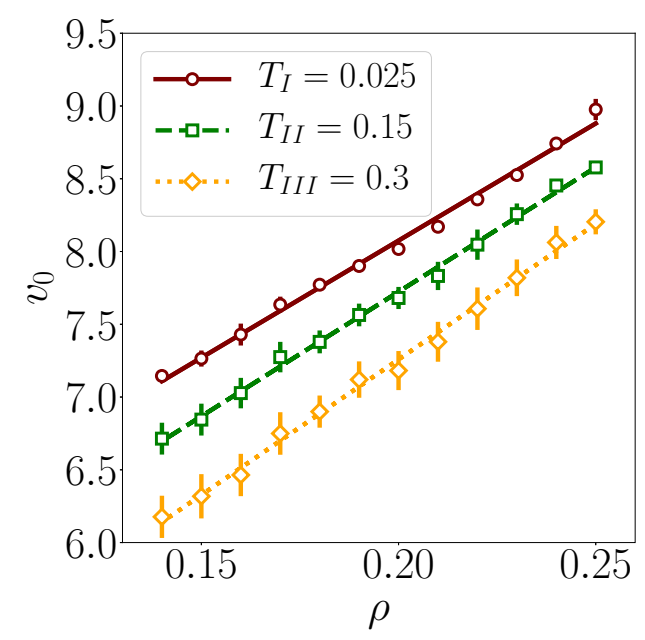

FIG. 6. Logistic midpoint as a function of the density for three temperatures, where the symbols are results of simulations and the lines are linear fits. 


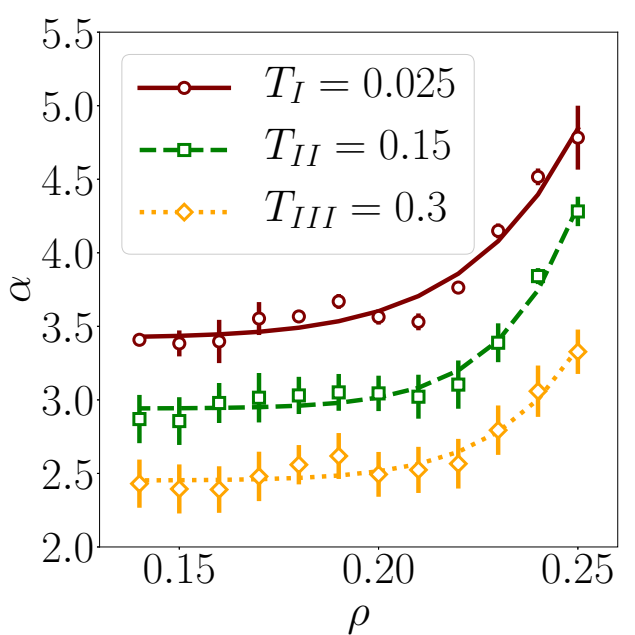

FIG. 7. Logistic steepness as a function of the density for three temperatures, where symbols represent results of simulations and lines are power-law curve fittings.

require a higher value of $v_{0}$ for the transition to global slip. The logistic steepness, $\alpha$, defined by Eq. (8), versus $\rho$ at temperatures $T=0.025,0.15$, and 0.3 is shown in Fig. 7. For low densities the value is almost constant, and for high density values the $\alpha$ value increases in power-law fashion.

Oscillatory behavior around a line is observed for both $v_{0}$ and $\alpha$ as a function of the density. The oscillatory behavior presented in Figs. 6 and 7 occurs at the same densities in both figures. The oscillations occur at densities from 0.25 to 0.20 and from 0.17 to 0.16 , where the number of layers shown in Fig. 4 changes from three to four and from four to five layers, respectively. So, even though the qualitative behavior of $P_{\mathrm{DD}}$ (Fig. 5) is also observed for LJ-like fluids, the small oscillations in $v_{0}$ and $\alpha$ versus the density indicate that the values assumed by $v_{0}$ and $\alpha$ are related to the unusual structures assumed by the waterlike fluid under confinement.

Next, we test whether the behavior of $v_{0}$ with the temperature, at a fixed density, is also affected by the number of layers. Figure 3 illustrates the behavior of the density versus the temperature. Three density regions are identified in this figure. In region I, $\rho=0.14,0.15$, and 0.16 , and the fluid forms five layers. In region II, $\rho=0.18$ and 0.19 , and the system is accommodated in four layers. In region III, $\rho=0.25$, $0.26,0.27$, and 0.28 , and three layers are formed. Figure 8 shows the behavior of the logistic midpoint as a function of the temperature for the densities in regions I (circles), II (squares), and III (diamonds) identified in Fig. 3. The transition velocity decreases linearly with the temperature at all densities analyzed. Densities in the same region (equal number of layers) have the same slope in the $v_{0}$-versus-temperature graph. For region I in Fig. 3 the slope is $b_{\mathrm{I}}=-3.09(2)$; for region II, $b_{\mathrm{II}}=-2.92(6)$; and for region III, $b_{\mathrm{III}}=-2.4(2)$.

Figure 9 shows the logistic steepness as a function of the temperature for densities in regions I (circles), II (squares), and III (diamonds). It decreases exponentially with the temperature, with an exponential fit $\left[\alpha=\alpha_{0} \exp (-\lambda T)\right]$. In region I the mean exponential decay coefficient is $\lambda_{\mathrm{I}}=0.84(2)$; in region II, $\lambda_{\mathrm{II}}=1.008(6)$; and in region III, $\lambda_{\mathrm{III}}=1.62(7)$. Differently

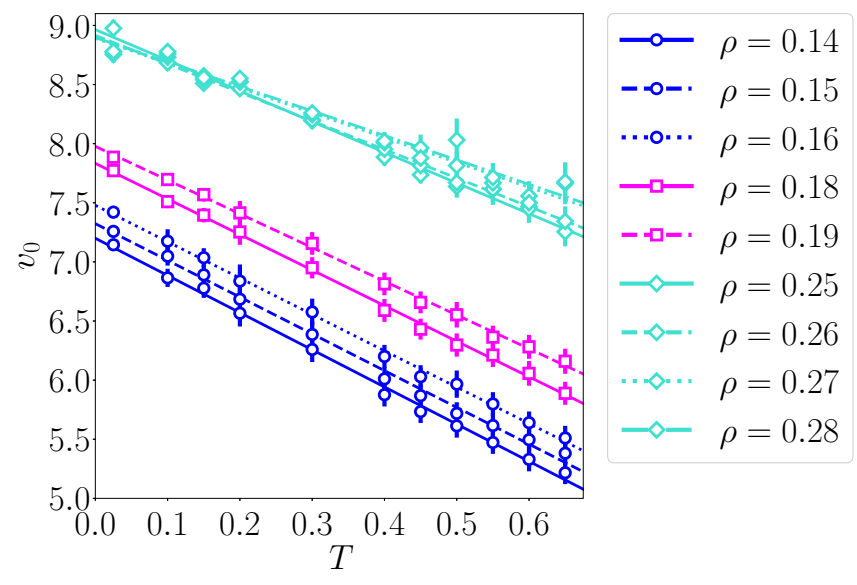

FIG. 8. Logistic midpoint as a function of the temperature for several densities, where lines are linear fits. Blue circles represent systems with five layers, magenta squares show systems with four layers, and turquoise diamonds show systems with three layers.

from the logistic midpoint, at high temperatures all curves collapse. In this case the $\alpha$ value is almost the same at all densities.

In order to understand how the transition to global slip depends on the number of layers, the density profile of the system is analyzed at different global densities and temperatures. At $\rho_{\mathrm{I}}=0.14$ and $T_{\mathrm{I}}=0.025$, as shown in Fig. 5, the no-slip condition is valid for velocities below 2 , the defect slip condition for $2<v_{x}<v_{0}^{\mathrm{I}}$, and the global slip condition for $v_{x}>v_{0}^{\mathrm{I}}$, where $v_{0}^{\mathrm{I}}=7.15(4)$. Figure 10 shows the dependence of the transversal density profile on $z$ for one bottom wall velocity under each boundary condition for $\rho_{\mathrm{I}}$ and $T_{\mathrm{I}}$ (see Fig. 3 for the location of this point in the density-versus-temperature phase diagram). The layering structure for different velocities under the no-slip condition $\left(v_{x}<2\right)$ is very similar to that in the $v_{x}=0$ case and exhibits layers without exchange of particles between them (solid line in Fig. 10). For velocities under the

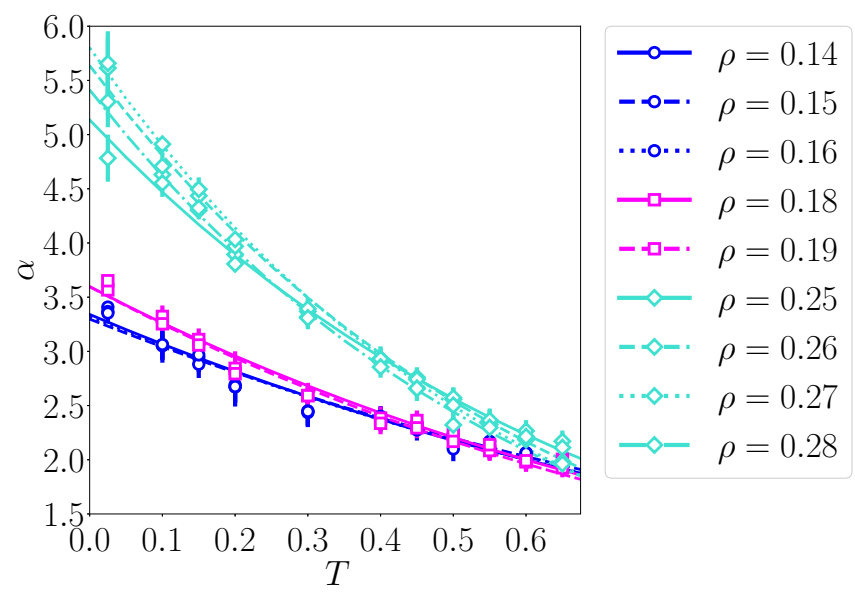

FIG. 9. Logistic steepness as a function of the temperature for several densities, where curves are exponential fits. Blue circles represent systems with five layers, magenta squares show systems with four layers, and turquoise diamonds show systems with three layers. 


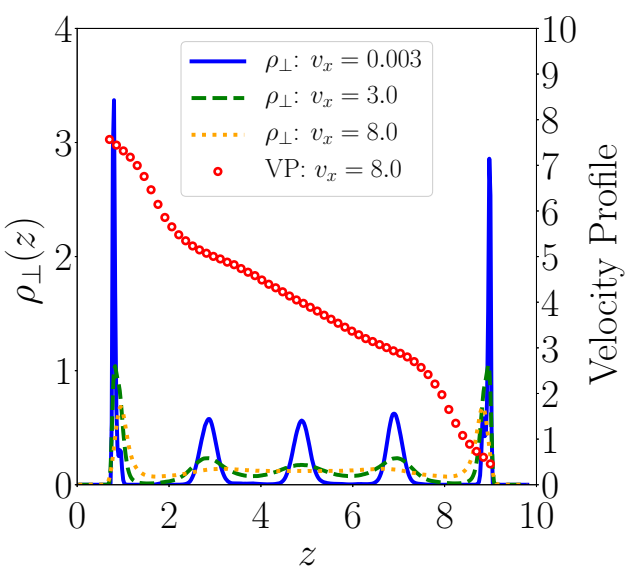

FIG. 10. Left axis: Transversal density profile at $\rho_{\mathrm{I}}=0.14$ and $T_{\mathrm{I}}=0.025$ for one bottom wall velocity under each boundary condition. Right axis: Fluid velocity profile at bottom wall velocity $v_{x}=8.0$.

defect slip condition the layers are present, but particles move between the layers (dashed line in Fig. 10). Under the global slip condition, at $v_{x}>v_{0}^{\mathrm{I}}$, the central layers are not present, and a uniform profile between the contact layers (dotted line in Fig. 10) is formed. At all densities studied at $T_{\mathrm{I}}=0.025 \mathrm{we}$ observe the same behavior as shown in Fig. 10. Figure 10 also shows the fluid velocity profile for $\rho_{\mathrm{I}}=0.14$ at $T_{\mathrm{I}}=0.025$ when the plate velocity is $v_{x}=8.0$ (circles). Even when the system is in the global slip regime, the velocity profile is not linear. This behavior is due to the structure in layers and indicates the difficulty of an accurate determination of the slip length.

For temperatures above the critical points in Fig. 3 the scenario is slightly different. In this range of temperatures no transition is present even in the $v_{x}=0$ case, and the increase in $v_{x}$ promotes a smooth change in $P_{\mathrm{DD}}$ as show in Fig. 11(a). In this case the increase in temperature promotes exchange of particles between the layers even in the no-slip case, as shown for $v_{x}=0.003$ (solid line) in Fig. 11(b). Consequently, defect
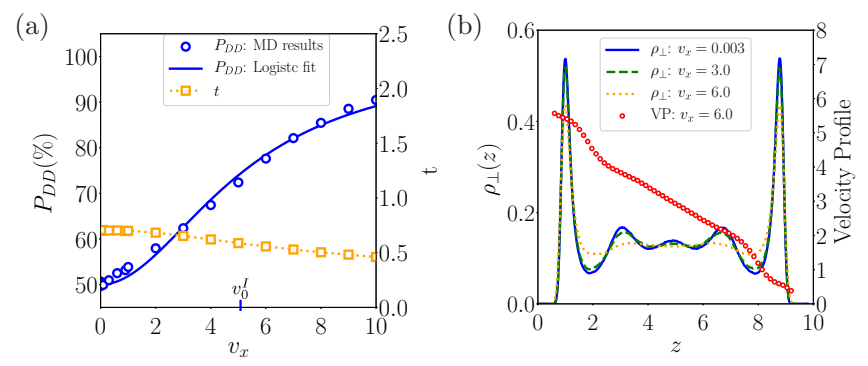

FIG. 11. (a) Left axis: Probability that a particle will move in the driven direction as a function of the bottom wall velocity. Blue circles represent results of simulations for $\rho_{\mathrm{I}}=0.14$ at $T=0.650$. The solid blue line is a logistic fit according to Eq. (8) with $v_{0}^{\mathrm{I}}=$ 5.2(1) and $\alpha^{\mathrm{I}}=1.97(9)$. Right axis: Translational order parameter as a function of the bottom wall velocity for $\rho_{\mathrm{I}}$ at $T=0.600$. (b) Left axis: Transversal density profile for $\rho_{\mathrm{I}}=0.14$ at $T=0.650$ for one bottom wall velocity under each boundary condition. Right axis: Fluid velocity profile at bottom wall velocity $v_{x}=6.0$.
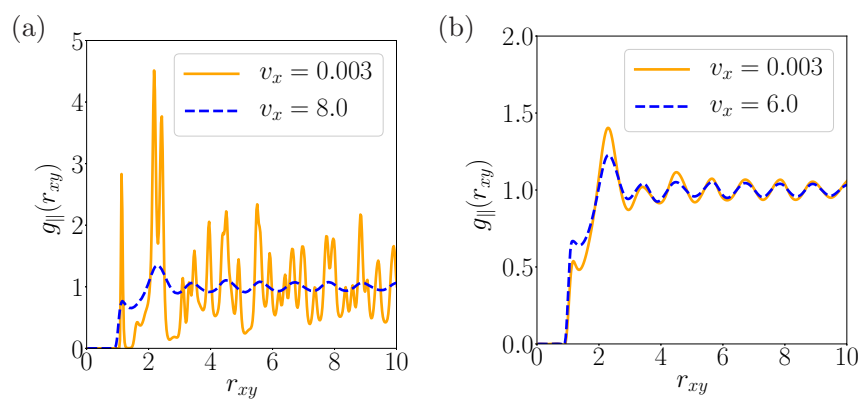

FIG. 12. Parallel radial distribution function of the contact layer for $\rho_{I}=0.14$ (a) at $T_{I}=0.025$ and (b) at $T=0.650$.

slip appears at very low bottom wall velocities [Fig. 11(a)], with no significant change in the transversal density profile [dashed line, $v_{x}=3.0$, in Fig. 11(b)]. At the global slip the central layers are destroyed, and the system presents a bulk profile between the contact layers [dotted line in Fig. 11(b)]. The velocity profile for high temperatures continues to show the nonlinear behavior observed at low temperatures [see Fig. 11(b)].

Since the behavior of the number of layers is affected by $v_{x}$ quite differently when the system is in the coexistence region compared with the supercritical region in Fig. 3, the response of the structure to the change in the velocity rate is analyzed in detail for both regions. Figures 5 and 11(a) show the translational order parameter (squares) as a function of the bottom wall velocity for $\rho_{I}=0.14$. The translational order parameter, $t$, decreases with increasing $v_{x}$; that is, the system becomes less structured with increasing shear level. In the coexistence region (Fig. 5), the decrease in the $t$ value is much more pronounced than in the supercritical region [Fig. 11(a)]. The difference in the structures at low vs high shear level is evident in the parallel radial distribution function.

Figure 12 shows the parallel radial distribution function of the contact layer at $\rho_{\mathrm{I}}=0.14$ and $T_{\mathrm{I}}=0.025$ [coexistence region; Fig. 12(a)] and $T=0.650$ [supercritical region; Fig. 12(b)] for low and high wall velocities. In the low-temperature case (coexistence region), the wall velocity leads to a transition from an amorphous phase to a liquid phase at $v_{x}>v_{0}^{\mathrm{I}}$ [Fig. 12(a)]. This behavior also was observed for $\rho_{\mathrm{II}}=0.18$ and $\rho_{\mathrm{III}}=0.25$ at $T_{\mathrm{I}}$ (coexistence region in Fig. 3). For temperatures above the critical points, the fluid is in the liquid phase regardless of the bottom wall velocities [Fig. 12(b)] and the $\alpha$ value is independent of the number of layers. This behavior also was observed for $\rho_{\mathrm{II}}$ and $\rho_{\mathrm{III}}$ at $T=0.650$.

\section{CONCLUSIONS}

In this work, we studied the dynamical behavior of a waterlike fluid under shear. As the wall speed increased a transition from defect to global slip conditions was observed.

We showed that defect slip appears due to an exchange of particles between the different fluid layers present in the confined waterlike fluid. The dynamics of this exchange is defined by the bottom wall velocity and the temperature. At low temperatures, the velocity of the bottom wall required for the defect slip to occur is high, while at high temperatures, this velocity is low. 
The bottom wall velocity necessary to promote the global slip condition depends on the density, temperature, and number of fluid layers. For a fixed density, the velocity of the bottom wall required for global slip to occur decreases linearly with increasing temperature, with a slope which depends on the number of fluid layers.

We also found that the transition from no slip to global slip is smoother at high temperatures where no phase transition is observed in the contact layer. In this situation the parameter $\alpha$ is independent of the density. At low temperatures the waterlike fluid presents different crystal-liquid phases, the parameter $\alpha$ presents high values dependent on the number of fluid layers, and the transition from no slip to global slip is less smooth.
Our findings are consistent with the hypothesis that the slip is dependent on the temperature, density, and shear rate. However, our work shows that although the slip is a dynamic phenomenon directly related to the contact layer, the behavior of the fluid between these layers is a determinant of the occurrence or nonoccurrence of the slip. Therefore, the anomalous dynamics of the confined waterlike fluid can be understood through the relation between the occurrence of slip at the liquid-solid interface and the anomalous thermodynamics and structure that water assumes under confinement.

\section{ACKNOWLEDGMENT}

We thank the Brazilian agencies CNPq and INCT-FCx for financial support.
[1] K. Watanabe, Y. Udagawa, and H. Udagawa, J. Fluid Mech. 381, 225 (1999).

[2] J. Baudry, E. Charlaix, A. Tonck, and D. Mazuyer, Langmuir 17, 5232 (2001).

[3] Y. Zhu and S. Granick, Phys. Rev. Lett. 87, 096105 (2001).

[4] Y. Zhu and S. Granick, Phys. Rev. Lett. 88, 106102 (2002).

[5] C. Cottin-Bizonne, B. Cross, A. Steinberger, and E. Charlaix, Phys. Rev. Lett. 94, 056102 (2005).

[6] C. Neto, D. R. Evans, E. Bonaccurso, H.-J. Butt, and V. S. J. Craig, Rep. Prog. Phys. 68, 2859 (2005).

[7] P. Joseph and P. Tabeling, Phys. Rev. E 71, 035303 (2005).

[8] L. Li, J. Mo, and Z. Li, Phys. Rev. E 90, 033003 (2014).

[9] L. Bocquet and J.-L. Barrat, Phys. Rev. E 49, 3079 (1994).

[10] O. I. Vinogradova, Langmuir 11, 2213 (1995).

[11] V. Sokhan, D. Nicholson, and N. Quirke, J. Chem. Phys. 115, 3878 (2001).

[12] L. Bocquet and J.-L. Barrat, Soft Matter 3, 685 (2007).

[13] A. Niavarani and N. V. Priezjev, Phys. Rev. E 81, 011606 (2010).

[14] R. Bhadauria, T. Sanghi, and N. R. Aluru, J. Chem. Phys. 143, 174702 (2015).

[15] E. Wagemann, E. Oyarzua, J. H. Walther, and H. A. Zambrano, Phys. Chem. Chem. Phys. 19, 8646 (2017).

[16] S. Lichter, A. Roxin, and S. Mandre, Phys. Rev. Lett. 93, 086001 (2004).

[17] S. Lichter, A. Martini, R. Q. Snurr, and Q. Wang, Phys. Rev. Lett. 98, 226001 (2007).

[18] A. Martini, H.-Y. Hsu, N. A. Patankar, and S. Lichter, Phys. Rev. Lett. 100, 206001 (2008).

[19] A. Martini, A. Roxin, R. Q. Snurr, Q. Wang, and S. Lichter, J. Fluid Mech. 600, 257 (2008).

[20] J.-T. Cheng and N. Giordano, Phys. Rev. E 65, 031206 (2002).

[21] H. Eslami and F. Muller-Plathe, J. Phys. Chem. B 114, 387 (2010).

[22] J. A. Thomas and A. J. H. McGaughey, Nano Lett. 8, 2788 (2008).

[23] A. Martini, Y. Liu, R. Q. Snurr, and Q. J. Wang, Tribol. Lett. 21, 217 (2006).

[24] D. C. Tretheway and C. D. Meinhart, Phys. Fluids 14, L9 (2002).

[25] E. Bonaccurso, M. Kappl, and H.-J. Butt, Phys. Rev. Lett. 88, 076103 (2002).

[26] C.-H. Choi, K. J. A. Westin, and K. S. Breuer, Phys. Fluids 15, 2897 (2003).
[27] O. I. Vinogradova, K. Koynov, A. Best, and F. Feuillebois, Phys. Rev. Lett. 102, 118302 (2009).

[28] Y. Xue, Y. Wu, X. Pei, H. Duan, Q. Xue, and F. Zhou, Langmuir 31, 226 (2014).

[29] M. Majumder, N. Chopra, R. Andrews, and B. J. Hinds, Nature 438, 44 (2005).

[30] J. K. Holt, H. G. Park, Y. Wang, M. Stadermann, A. B. Artyukhin, C. P. Grigoropoulos, A. Noy, and O. Bakajin, Science 312, 1034 (2006).

[31] X. Qin, Q. Yuan, Y. Zhao, S. Xie, and Z. Liu, Nano Lett. 11, 2173 (2011).

[32] P. Ternes, A. Mendoza-Coto, and E. Salcedo, J. Chem. Phys. 147, 034510 (2017).

[33] S. K. Kannam, B. D. Todd, J. S. Hansen, and P. J. Daivis, J. Chem. Phys. 136, 024705 (2012).

[34] J. Koplik, J. R. Banavar, and J. F. Willemsen, Phys. Fluids A 1, 781 (1989).

[35] P. A. Thompson and M. O. Robbins, Phys. Rev. A 41, 6830 (1990).

[36] P. A. Thompson and S. M. Troian, Nature 389, 360 (1997).

[37] J.-L. Barrat and L. Bocquet, Phys. Rev. Lett. 82, 4671 (1999).

[38] R. Pit, H. Hervet, and L. Leger, Phys. Rev. Lett. 85, 980 (2000).

[39] M. Cieplak, J. Koplik, and J. R. Banavar, Phys. Rev. Lett. 86, 803 (2001).

[40] L. Joly, C. Ybert, and L. Bocquet, Phys. Rev. Lett. 96, 046101 (2006).

[41] N. V. Priezjev and S. M. Troian, J. Fluid Mech. 554, 25 (2006).

[42] N. V. Priezjev, Phys. Rev. E 75, 051605 (2007).

[43] P. A. Netz, F. W. Starr, H. E. Stanley, and M. C. Barbosa, J. Chem. Phys. 115, 344 (2001).

[44] L. Xu, P. Kumar, S. V. Buldyrev, S.-H. Chen, P. H. Poole, F. Sciortino, and H. E. Stanley, Proc. Natl. Acad. Sci. USA 102, 16558 (2005).

[45] L. Xu, S. V. Buldyrev, C. A. Angell, and H. E. Stanley, Phys. Rev. E 74, 031108 (2006).

[46] A. B. de Oliveira, P. A. Netz, T. Colla, and M. C. Barbosa, J. Chem. Phys. 125, 124503 (2006).

[47] A. B. de Oliveira, P. A. Netz, T. Colla, and M. C. Barbosa, J. Chem. Phys. 124, 084505 (2006).

[48] N. M. Barraz, Jr., E. Salcedo, and M. C. Barbosa, J. Chem. Phys. 131, 094504 (2009). 
[49] J. R. Bordin, L. B. Krott, and M. C. Barbosa, J. Chem. Phys. 141, 144502 (2014).

[50] J. R. Bordin, L. B. Krott, and M. C. Barbosa, J. Phys. Chem. C 118, 9497 (2014).

[51] C. Gavazzoni, N. Giovambattista, P. A. Netz, and M. C. Barbosa, J. Chem. Phys. 146, 234509 (2017).

[52] M. Neek-Amal, F. M. Peeters, I. V. Grigorieva, and A. K. Geim, ACS Nano 10, 3685 (2016).

[53] P. Gallo, M. Rovere, and S.-H. Chen, J. Phys.: Condens. Matter 22, 284102 (2010).

[54] A. B. Farimani and N. R. Aluru, J. Phys. Chem. C 120, 23763 (2016).

[55] M. De Marzio, G. Camisasca, M. M. Conde, M. Rovere, and P. Gallo, J. Chem. Phys. 146, 084505 (2017).

[56] M. Sega, M. Sbragaglia, L. Biferale, and S. Succi, Soft Matter 9, 8526 (2013).

[57] J. E. Jones, Proc. R. Soc. London A 106, 463 (1924).

[58] J. E. Lennard-Jones, Proc. Phys. Soc. 43, 461 (1931).
[59] T. Head-Gordon and F. H. Stillinger, J. Chem. Phys. 98, 3313 (1993).

[60] F. H. Stillinger and T. Head-Gordon, Phys. Rev. E 47, 2484 (1993).

[61] J. D. Weeks, D. Chandler, and H. C. Andersen, J. Chem. Phys. 54, 5237 (1971).

[62] D. Frenkel and B. Smit, Understanding Molecular Simulation, 1st ed. (Academic, San Diego, CA, 1996).

[63] M. P. Allen and D. J. Tildesley, Computer Simulations of Liquids, 1st ed. (Clarendon, Oxford, UK, 1987).

[64] P. Kumar, S. V. Buldyrev, F. W. Starr, N. Giovambattista, and H. E. Stanley, Phys. Rev. E 72, 051503 (2005).

[65] P. Kumar, F. W. Starr, S. V. Buldyrev, and H. E. Stanley, Phys. Rev. E 75, 011202 (2007).

[66] W. G. Hoover, Phys. Rev. A 31, 1695 (1985).

[67] W. G. Hoover, Phys. Rev. A 34, 2499 (1986).

[68] R. Zangi and S. A. Rice, Phys. Rev. E 61, 660 (2000).

[69] J. R. Errington and P. G. Debenedetti, Nature 409, 318 (2001). 